\title{
BMJ Open Study protocol: retrospectively mining multisite clinical data to presymptomatically predict seizure onset for individual patients with Sturge-Weber
}

\author{
Pooja Vedmurthy (D) , ${ }^{1,2}$ Anna L R Pinto, ${ }^{3}$ Doris D M Lin, ${ }^{4}$ Anne M Comi, ${ }^{1,2,5}$ \\ Yangming $\mathrm{Ou},{ }^{6,7,8} \mathrm{BCH}-\mathrm{KKI}$ SWS Pre-symptomatic Biomarker Working Group
}

To cite: Vedmurthy $P$, Pinto ALR, Lin DDM, et al. Study protocol: retrospectively mining multisite clinical data to presymptomatically predict seizure onset for individual patients with Sturge-Weber. BMJ Open 2022;12:e053103. doi:10.1136/ bmjopen-2021-053103

- Prepublication history for this paper is available online. To view these files, please visit the journal online (http://dx.doi. org/10.1136/bmjopen-2021053103).

PV and ALRP are joint first authors.

$A M C$ and $Y 0$ are joint senior authors.

Received 05 May 2021 Accepted 13 January 2022

D Check for updates

(c) Author(s) (or their employer(s)) 2022. Re-use permitted under CC BY-NC. No commercial re-use. See rights and permissions. Published by BMJ.

For numbered affiliations see end of article.

Correspondence to Dr Yangming $\mathrm{Ou}$; Yangming.0u@childrens. harvard.edu

\section{ABSTRACT}

Introduction Secondary analysis of hospital-hosted clinical data can save time and cost compared with prospective clinical trials for neuroimaging biomarker development. We present such a study for Sturge-Weber syndrome (SWS), a rare neurovascular disorder that affects 1 in 20000-50 000 newborns. Children with SWS are at risk for developing neurocognitive deficit by school age. A critical period for early intervention is before 2 years of age, but early diagnostic and prognostic biomarkers are lacking. We aim to retrospectively mine clinical data for SWS at two national centres to develop presymptomatic biomarkers.

Methods and analysis We will retrospectively collect clinical, MRI and neurocognitive outcome data for patients with SWS who underwent brain MRI before 2 years of age at two national SWS care centres. Expert review of clinical records and MRI quality control will be used to refine the cohort. The merged multisite data will be used to develop algorithms for abnormality detection, lesion-symptom mapping to identify neural substrate and machine learning to predict individual outcomes (presence or absence of seizures) by 2 years of age. Presymptomatic treatment in $0-2$ years and before seizure onset may delay or prevent the onset of seizures by 2 years of age, and thereby improve neurocognitive outcomes. The proposed work, if successful, will be one of the largest and most comprehensive multisite databases for the presymptomatic phase of this rare disease.

Ethics and dissemination This study involves human participants and was approved by Boston Children's Hospital Institutional Review Board: IRB-P00014482 and IRB-P00025916 Johns Hopkins School of Medicine Institutional Review Board: NA_00043846. Participants gave informed consent to participate in the study before taking part. The Institutional Review Boards at Kennedy Krieger Institute and Boston Children's Hospital approval have been obtained at each site to retrospectively study this data. Results will be disseminated by presentations, publication and sharing of algorithms generated.

\section{INTRODUCTION}

About 1/1000 infants are born with port-wine birthmarks (PWB) on the face or neck. Of

\section{Strengths and limitations of this study}

- We will retrospectively gather multisite neuroimaging and comprehensive data from $>100$ subjects with Sturge-Weber syndrome, a rare disease, compared with prior single-site studies with typically only a dozen or so patients.

- This will be the first rigorous multisite neuroimaging biomarker study in the presymptomatic phase of Sturge-Weber syndrome, compared with most studies focusing on the postsymptomatic phase.

-We will develop and use statistically rigorous lesionsymptom mapping to predict neurocognitive outcomes in Sturge-Weber syndrome and rigorously test whether combining clinical and MRI information outperforms brain MRI alone in outcome prediction.

- Heterogenous brain MRI protocols (scanner, vendor, scanning site, age at MRI, etc) will be used; however, this heterogeneity increases the generalisability of the results.

those infants, 5\% develop a vascular neurocutaneous disorder called Sturge-Weber syndrome (SWS). ${ }^{1}$ This makes SWS patients 1 in 20000 to 1 in 50000 newborns, and, therefore, a rare disease. ${ }^{23}$ SWS is usually caused by an activating somatic mutation in the gene $G N A Q$, encoding the $\mathrm{G}_{\mathrm{q}}$ subunit in the affected tissue. ${ }^{1}$ Diagnosis is based on the classic PWB at birth and vascular findings in brain MRI that are routinely acquired during infancy. The disease is not commonly fatal but is incurable. ${ }^{45}$ Neurocognitive impairment by school age (6-10 years of age) is a primary issue in SWS.

A landmark event in the progression of SWS is the presence or absence of seizures by 2 years of age (figure 1). About $15 \%$ of patients with SWS do not develop seizures by 2 years and they often enjoy good school-age 


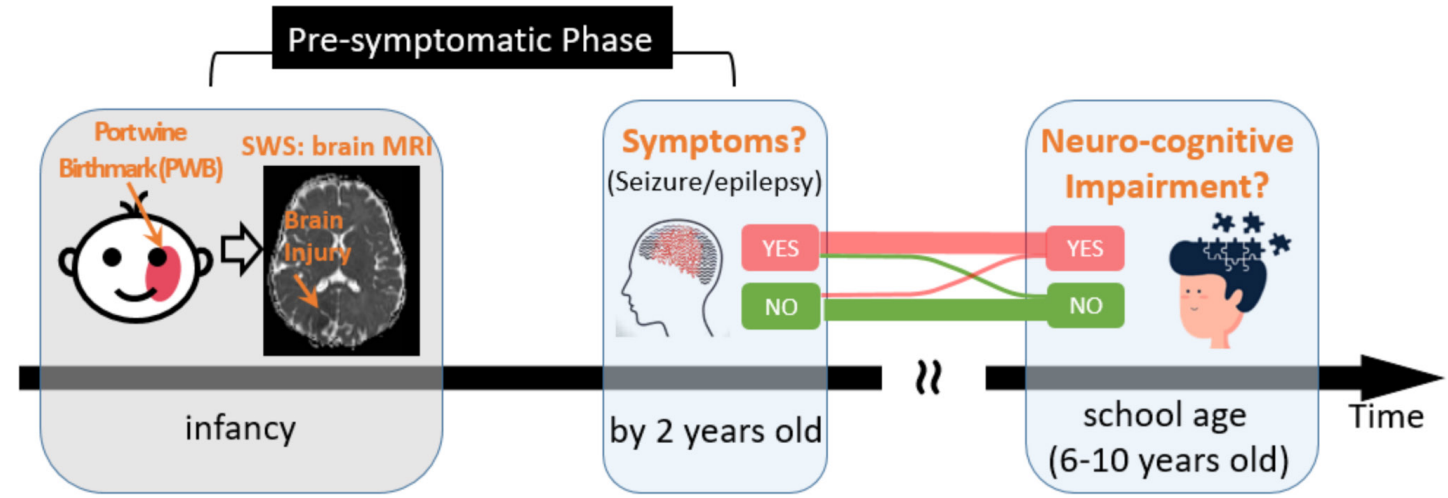

Figure 1 Need for the early prediction of risk to develop epilepsy/seizure symptoms by 2 years of age among patients with SWS in the 'pre-symptomatic phase'. SWS, Sturge-Weber syndrome.

neurocognitive outcomes ${ }^{6} 7$ Because of this, a critical time window to treat or intervene in patients with SWS is during infancy up to 2 years of age, the so-called presymptomatic phase. In this phase, very early treatment with antiepilepsy drugs (AEDs, such as levetiracetam, with or without low-dose aspirin $)^{8-10}$ may delay or even avoid seizure symptoms by 2 years of age and thus improve school-age outcomes. ${ }^{67}$ The evidence of the early onset of seizures resulting in worse neurologic outcome ${ }^{6}$ has led to a demand for early diagnosis and aggressive intervention for those at increased risk of developing seizures.

However, two questions remain open. For one, how to accurately identify who is at risk to develop the seizure/ epilepsy symptoms by 2 years of age? These at-risk patients should be the ideal target patients to be included in future trials for testing AEDs in the critical 'presymptomatic' time window. For the other, what are the underlying neuroanatomic mechanisms that drive infants with SWS to develop epilepsy/seizure symptoms before 2 years of age? The neuromechanisms should be the targets to design the next-generation AEDs or other presymptomatic treatment strategies. ${ }^{411}$

Infant brain MRI is suggested by recent expert consensus $\left(2018^{4} ; 2019\right)^{5}$ in the field to address these two open questions. This is because infant brain MRI is routinely acquired during the clinical care of patients with SWS and is non-invasive. Besides, MRI can probe 3D neuroanatomy, with both diagnostic value (commonly seen presymptomatic brain abnormalities including leptomeningeal, parenchymal and choroidal capillary venous malformations with congenitally abnormal and ectatic vessels) ${ }^{12}$ and prognostic value (abnormal neuronal excitation, ${ }^{13}$ transient diffusion abnormalities ${ }^{14}{ }^{15}$ and bilateral hemispheric injuries ${ }^{1}$ as seen in MRI may suggest an adverse outcome). However, these findings were based on single-site data, small sample sizes (single-digit or only a dozen or so). Moreover, expert interpretations of MRIs are largely qualitative or descriptive and may not be replicated across sites. This issue is further complicated by the limited clinical radiology expertise on this rare disease, especially in less developed regions or countries. ${ }^{416}$
To address these issues, this study aims to collect a relatively large multisite cohort for this rare disease and develop machine learning biomarkers. Existing MRI studies for SWS usually had $\leq 15^{517-19}$ or dozens ${ }^{11162021}$ of patients with SWS in single sites. We aim to merge existing clinical data from Boston Children's Hospital (BCH) and Kennedy Krieger Institute (KKI) to achieve an sample size at least five times bigger. Existing MRI studies for SWS mostly used expert reviews. We will leverage MRI analytics and machine learning algorithms, which have had success in characterising subtle yet complex injury patterns $^{22}{ }^{23}$ and in predicting outcomes for stroke, ${ }^{24}$ tumour, ${ }^{25}$ autism ${ }^{26}$ and other neurologic diseases, ${ }^{27}$ but have not yet been used in SWS. Our hypothesis is that machine learning-driven MRI and clinical biomarkers can predict the onset or absence of seizure symptoms by 2 years at a sensitivity and specificity higher than random guess (ie, 50\%). Setting the random guess as the bar is because the lack of such a presymptomatic biomarker for SWS.

\section{METHODS AND ANALYSIS Overview}

This retrospective study was approved by institutional review boards at BCH and KKI. Written consent was waived for the retrospective analysis of existing data that are anonymised. Figure 2 outlines the major components of our study. We plan to start the study in April or December of 2022 (pending funding from National Institute of Health $(\mathrm{NIH}))$. The study period will be for $\sim 2$ years.

\section{Part 1. Retrospective data acquisition}

\section{Find candidate patients}

We plan to collect data for all patients who (a) were born between 2009 and 2020, (b) were under 2 years of age at the first MRI and (c) had SWS brain involvement confirmed by contrast-enhanced MRI before 2 years of age.

Patients will be primarily identified by clinical registries, in the neurology departments from patients cared by Dr. Pinto at BCH and Dr. Comi at KKI. 
Part 1. Retrospective Data Collection

1.1. Find Candidate Patients

1.2. Query and fetch clinical data

1.3. Gather MRI data and quality control

1.4. Manage Data

Figure 2 Outline of our study protocol.
Part 2. Identify Patterns

(population analysis)

2.1. MRI pre-processing

2.2. Abnormality Detection

2.3. Mapping brain injury patterns to outcome
Part 3. Outcome Prediction

(individual precision)

3.1. Traditional machine learning models

3.2. Deep learning outcome prediction

3.3. Integrate MRI and non-MRI information
A secondary source will be the hospital-wide database. We will search clinical database and picture achieving and communication system (PACS) imaging systems by the ICD codes for SWS (table 1). Candidates missed in the clinical registry will be further vetted by their clinical records to confirm eligibility to be included in our database.

\section{Query and fetch clinical data}

We will review patients' electronic health records (EHRs) and gathered the following clinical variables.

- Demographics: sex, the extent of brain involvement, the extent of PWB (bilateral, unilateral in the left or right side), age at first MRI, year of MRI, age at electroencephalogram (EEG) and clinical result, year of birth and family history of epilepsy

- Treatment: whether the patient was treated presymptomatically (ie, before the first onset of seizures).

- Outcome by 2 years: the primary neurological outcome will be the presence or absence of seizure onset by 2 years, and the age at seizure onset if present. The secondary outcome is the SWS neuroscore (including seizure score). The neuroscore is a scale that measures seizure frequency, hemiparesis, visual field cut and cognitive function (points range from 0 to 15 , with lower scores indicating better neurologic status. ${ }^{28-31}$ Patients treated presymptomatically were assigned a seizure score of 0 at the time of treatment initiation. Subjects with a seizure score of 0 at 2 years of age are patients who have achieved 2 years of age without seizure onset.

Gather brain MRI data and quality control

We will use patients' medical record numbers to search and download their brain MRIs in the clinical PACS. The Computational Health Research Integration System (ChRIS) platform offers this function, ${ }^{32}$ similar to other retrospective paediatric brain MRI projects at $\mathrm{BCH} .{ }^{27} 33$ $\mathrm{KKI}$ also hosts the $\mathrm{i} 2 \mathrm{~b} 2^{34}$ platform for this function.

MRI sequences to be downloaded include: T1-weighted (T1w; spin echo vs 3D gradient echo), T2-weighted (T2w) spin echo, susceptibility-weighted imaging (SWI) including the phase and magnitude images, or T2*-weighted gradient echo, fluid-attenuated inversion recovery (FLAIR), diffusion-weighted imaging or diffusion tensor imaging (DTI) and postgadolinium-contrast T1w (spin echo or 3D gradient-echo T1 contrast (T1c) images).

For quality control, MRIs will be visually reviewed by imaging experts (Dr. Ou for BCH data and Dr. Lin for KKI data). MRIs with severe motion, artefacts or part of the brain missing will be discarded.

For the kept MRIs, we will record scanner vendors, scanner models, field of strength, in addition to the available sequences among those listed above.

\section{Manage data}

We will use REDCap ${ }^{35}$ to manually enter and maintain clinical variables from EHR. REDCap is Health Insurance Portability and Accountability Act (HIPAA)-complicant, secure and user-friendly and has been used in similar multisite database by this team. ${ }^{27}$

\section{Part 2. Identify presymptomatic injury patterns for seizure outcome (population analysis) \\ MRI preprocessing}

Each patient's T1w brain MRI will undergo our extensively validated brain MRI analysis pipeline. Steps include $\mathrm{N} 4$ bias correction, ${ }^{36}$ field of view normalisation, ${ }^{37}$ multiatlas skull stripping, ${ }^{38}$ atlas-based regional segmentation ${ }^{39}$ as adapted and validated in infants $0-2$ years of age. ${ }^{38} 4041$ The computed brain mask in T1w MRI will automatically skull strip other MRI sequences when rigidly aligned with the T1w MRI. ${ }^{2}$ To compare across patients, their T1w

Table 1 ICD-9/10 codes for SWS as a secondary source to query candidate patients beyond the clinical registry as the primary source

\begin{tabular}{llll}
\hline ICD-9 & Meaning & ICD-10 & Meaning \\
\hline 759.6 & Other hamartoses, not elsewhere classified & Q85.8 & $\begin{array}{l}\text { Other phakomatoses, not } \\
\text { elsewhere classified }\end{array}$ \\
$\mathbf{7 5 7 . 3 2}$ & $\begin{array}{l}\text { Angiomatosis } \\
\text { 759.6 }\end{array}$ & $\mathbf{Q 8 5 . 0 2}$ & Other congenital malformations \\
& $\begin{array}{l}\text { Sturge (-Weber) (-Dimitri) (encephalocutaneous } \\
\text { angiomatosis) }\end{array}$ & & \\
\hline
\end{tabular}

SWS, Sturge-Weber syndrome. 


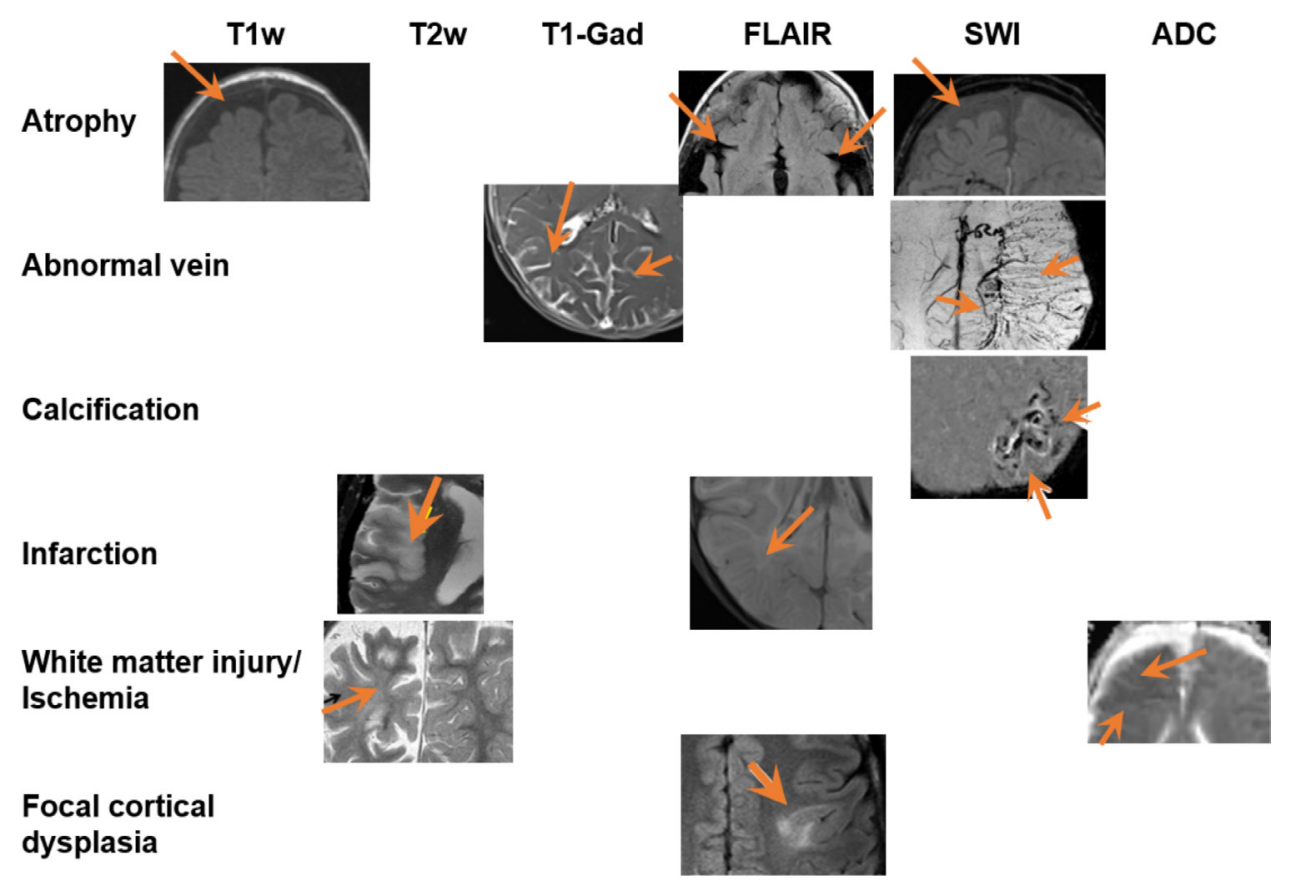

Figure 3 Typical abnormalities (different rows) found in the brain MRI of patient with SWS, in multiple MRI sequences (different columns). Different figure panels are from different patients. Orange arrows point out the abnormal regions. ADC, apparent diffusion coefficient; FLAIR, fluid-attenuated inversion recovery; SWI, susceptibility-weighted imaging; SWS, Sturge-Weber syndrome.

MRIs will be non-rigidly aligned into our atlas space ${ }^{33}$ by our validated ${ }^{42}$ and public deformable image registration algorithm. ${ }^{43}$ DTIs will be used to derive the fractional anisotropy (FA) and apparent diffusion coefficient (ADC) maps, after motion correction and tensor reconstruction using Functional Magnetic Resonance Imaging of the Brain Software Library (FSL) ${ }^{44}$ by factoring out the effects of scanners and sites, while controlling for other covariates such as sex, age, race, etc. We will use the validated ${ }^{45}$ ComBat tool to harmonise MRI metrics extracted from multisite data. ${ }^{46}$

\section{Abnormality detection}

Figure 3 shows different types of abnormalities associated with SWS that manifest in multiparametric brain MRI:

- atrophy, infarction and focal cortical dysplasia or polymicrogyria are often observed in T1 $\mathbf{w}^{11} 14213047-49$ and T2 $\mathbf{w}^{50-52}$, FLAIR ${ }^{50-52}$ and SWI ${ }^{53}$;

- abnormal veins (leptomeningeal venous malformation, enlarged deep medullary and ependymal veins) are often observed in $\mathrm{T} 1 \mathrm{w}$ with Gadolinium-contrast enhancement (T1-Gad ${ }^{125-55}$ ), and $\mathbf{S W I}^{5456}$;

- calcification is best visualised on $\mathrm{SWI}^{53}$ );

- abnormal diffusion, accelerated myelination or ischaemic injury are often observed in ADC map, ${ }^{57-59}$ a parameter map from diffusion MRI sequences; and

- damages in the white matter microstructures or disruptions in fibre tracts are often observed inFA map ${ }^{60-62}$ of the DTI.

To automate the abnormality detection, we will extend our recently-developed algorithm that quantifies normal and detect abnormalities as outliers to normal.
The first major component in this algorithm is to quantify normal through constructing age-specific normal brain atlases. The atlases will quantify the normal range of signals (mean and SD) at each voxel in a normal control cohort. We recently developed age-specific normal ADC atlases, based on ADC maps from 201 normal participants who were scanned during 0-6 years of age, divided into 10 age groups (first 2 weeks, every quarter in the first year, then every year till 6 years). ${ }^{33}$ The atlases in each age group quantified voxel-wise normal range of ADC values, and characterised regional and hemispheric differences in early-childhood brain development patterns. ${ }^{40}$ The atlases are unbiased to any subject's anatomy, ${ }^{6364}$ based on our extensively-validated ${ }^{42}$ group-wise deformable image registration algorithm. ${ }^{43}$ We plan to build such age-specific atlases for each MRI sequence (T1w, T2w, FLAIR, SWI, ADC), based on our recently-gathered $>200$ multi-parametric brain MRIs on normal participants. These normal brain MRIs are from published data acquired from Massachusetts General Hospital and BCH (see references for more details on demographics and imaging protocols) $)^{3340}$ and they are distinctive from the SWS patient data we plan to merge from KKI and $\mathrm{BCH}$.

The second step is to detect abnormalities. This will be done by converting the patient's MRI signal in each sequence into a $\mathrm{Z}$ score map. The $\mathrm{Z}$ map will quantify voxel-wise deviation (eg, how many SD away) from the normal mean intensity at the corresponding voxel in the age-matched atlas of the same sequence. We recently used this strategy and successfully identified pre-symptomatic ischaemia lesions in ADC maps of 7 SWS patients. ${ }^{59}$ 
Another study used a similar strategy on T1w and T2w images (after histogram normalisation) and identified white matter abnormalities in preterm infant's brain MRIs. ${ }^{65}$ We will do this for each MRI sequence, generating $\mathrm{Z}_{\mathrm{T} 1}, \mathrm{Z}_{\mathrm{T} 2}, \mathrm{Z}_{\mathrm{FLAIR}}, \mathrm{Z}_{\mathrm{SWI}}$ and $\mathrm{Z}_{\mathrm{ADC}}$ maps, and we will call abnormalities when $Z$ values are lower than -2 .

Accuracy for abnormality detection will be quantified by calculating the Dice overlap, sensitivity and specificity compared with expert-annotated abnormality regions. However, expert annotations of exact abnormality boundary in patients with SWS will be subjected to intrareader/inter-reader variability. Therefore, an alternative approach is to indirectly evaluate the accuracy of abnormality detection in the context of pattern analysis and individual outcome prediction (later sections).

\section{Mapping brain injury patterns to outcome}

We will focus on the group differences between those who had MRI and no symptom till 2 years (group A) and those whose seizure occurred after MRI and before 2 years (group B). The postsymptomatic patients (first MRI occurred after seizures but before 2 years of age) will form a group $\mathrm{C}$ that serves as a validation cohort.

The key question is the 'neural substrate' underlying outcome-namely, patterns of brain injuries in the presymptomatic MRI that underpin the subsequent development of seizure by 2 years? This maps to group analysis between group A and group B.

We will use the lesion-symptom mapping (LSM) framework $^{6667}$ to identify the neural substrate of the outcomes (seizure onset or not) by 2 years. To reduce false positives, we will control for patient sex, age at MRI and whole-brain abnormality volume as covariates. ${ }^{68} 69$ Three versions of LSM models will be used and compared. Voxel-wise LSM (V-LSM) compares whether the presence or absence of abnormality at each voxel is statistically associated with the binary outcomes across patients. ${ }^{68}$ A limitation of V-LSM is that it overlooks the potential interaction among voxels. To address this, multivariate LSM (M-LSM) jointly considers voxels and voxel clusters and uses multivariate machine learning (support vector machine (SVM)) to find a subset of voxels that jointly associate with outcomes. ${ }^{70}{ }^{71}$ A potential limitation of M-LSM is that the autoselected voxel clusters may not have anatomic explanations. To address this, the latest generation of LSM, known as connectome-based LSM or C-LSM, ${ }^{72-74}$ builds on findings in NIH's human connectome project ${ }^{75-77}$ and NIH's Brain Research Through Advancing Innovative Neurotechnologies (BRAIN) initiative ${ }^{77}$ and quantifies which fibre bundles or functional brain circuits, if injured, are associated with outcomes. ${ }^{72}$ The assumption is that patients may differ in exact anatomic locations that are injured, but their injuries may intersect with the same fibre bundles or brain circuit, which will explain why they experience similar outcomes.

LSM successfully identified neural substrates of survival and language outcomes after adult brain tumor ${ }^{78}$; LSM identified neural substrates of language,$^{79-81}$ motor, ${ }^{82}$ word comprehension, ${ }^{83}$ voice recognition, ${ }^{84}$ attention, ${ }^{85}$ computational ability $^{82}$ and somatosensory outcomes ${ }^{86}$ after adult stroke lesions, etc. These studies used one LSM model, not three, and mostly V-LSM, the basic LSM model. We plan to use all three LSM models, with different assumptions, for the first time in SWS, based on the identified abnormality regions from the previous task (abnormality detection).

We will use two methods to validate the identified neural substrate for the outcome. One indirect validation will be by comparison with group C (postsymptomatic cohort). We hypothesise that the injury patterns that differentiate between group A and group B are the early and subtle stage of abnormalities that will also manifest, to a more severe extent, in group $\mathrm{C}$. The other validation is a direct quantification of the accuracy in individual predictions, as described next.

\section{Part 3. Outcome prediction (individual precision)}

The previous part 2 focuses on the group contrast. However, patients in the same outcome group are different in their clinical and MRI characteristics. The key question in this part 3 is: how to use the presymptomatic brain MRI to predict which patient is at risk to develop seizures after MRI and by 2 years? This is individual prediction.

\section{Traditional machine learning models}

$\mathrm{SVM}^{87} 88$ or random forest (RF), ${ }^{89} 90$ represent each patient by a feature vector. One set of features will come from the presence or absence $(1 / 0)$ of abnormality in the key neuroanatomic regions as identified by V-LSM, M-LSM and C-LSM neural substrate pattern analysis. This set of features assumes that the spatial patterns of abnormalities can infer outcome. Our second set of features will be imaging appearance features, as listed in table 2 . We will concatenate features in neural substrates (spatial features) and imaging appearance features into a long feature vector to represent a patient. We will use our iterative forward inclusion and backward elimination (iFIBE) feature selection algorithm to automatically identify the subset of features that optimally predict outcomes. ${ }^{43}$ 91-94 Here, optimality is defined as the sensitivity, specificity and area under the receiver operating characteristic curve (AUC) in $\mathrm{k}$-fold (we will choose $\mathrm{k}=5$ ) cross validations.

Not all features are equally useful. Some features may be redundant. To select the best feature combinations, we developed and implemented into software an iFIBE feature selection tool. ${ }^{43} 91$ Usually, iFIBE automatically selects 5-70 features out of hundreds to thousands of features. It automatically decides how many features to select, based on tasks. It starts from the single most predictive feature (by AUC in cross-validation) and iteratively adds features that increase the AUC than adding any other feature. When no more features can be added that further improves AUC, the algorithm removes features from the selected subset, one at a time, such that the removed feature will increase the AUC of the 
Table 2 MRI appearance features characterising abnormalities for outcome prediction.

\begin{tabular}{|c|c|}
\hline Categories & Details of features \\
\hline $\begin{array}{l}\text { I.Anatomy of } \\
\text { abnormalities }\end{array}$ & $\begin{array}{l}\text { I.1. Mass centre in standard atlas space; } \\
\text { I.2. Percentage of the whole-brain volume and the volume of each of the } 61 \text { auto-segmented brain } \\
\text { structures being injured }{ }^{39} 41 \text {; } \\
\text { l.3. Ratios of volumetric injury in the same brain structures between the left and right hemisphere; } \\
\text { l.4. Percentage and distribution of abnormalities in } 28 \text { major fibre tracts as defined in the JHU atlas. }{ }^{145}\end{array}$ \\
\hline $\begin{array}{l}\text { II. Geometry of } \\
\text { abnormalities }\end{array}$ & $\begin{array}{l}\text { II.1. Volume of abnormal regions; } \\
\text { II.2. Maximum diameter along different orthogonal directions, maximum surface of abnormal regions, } \\
\text { geometric compactness, spherecity, surface-to-volume ratio in the abnormal regions. }\end{array}$ \\
\hline $\begin{array}{l}\text { III. Heterogeneity of } \\
\text { abnormalities }\end{array}$ & $\begin{array}{l}\text { III.1. Histogram analysis }\left(0,25,50,75 \text { and } 100 \text {-percentile) of } T 1 w, T 2 w, T 1-G a d, F L A I R, A D C, S W I, Z_{T 1 w}\right. \\
Z_{\text {T2w }}, Z_{F L A I R}, Z_{A D C}, Z_{S W I} \text { signal values within the abnormal regions; } \\
\text { III.2. Skewness (asymmetry), kurtosis (flatness), and randomness (entropy, SD) of T1w, T2w, T1-Gad, } \\
\text { FLAIR, ADC, SWI, } Z_{T 1 w}, Z_{T 2 w}, Z_{F L A I R}, Z_{A D C}, Z_{S W I} \text { signal values within abnormal regions; }\end{array}$ \\
\hline $\begin{array}{l}\text { IV. Texture of } \\
\text { abnormalities }\end{array}$ & $\begin{array}{l}\text { IV.1. Gray-level co-occurrence matrix features and gray-level run-length matrix of T1w, T2w, T1-Gad, } \\
\text { FLAIR, ADC, SWI, } Z_{\mathrm{T} 1 \mathrm{w}}, \mathrm{Z}_{\mathrm{T} 2 \mathrm{w}}, \mathrm{Z}_{\mathrm{T} \text {-Gad }}, \mathrm{Z}_{\mathrm{FLAIR}}, \mathrm{Z}_{\mathrm{ADC}}, \mathrm{Z}_{\mathrm{SWl}} \text { signal values within abnormal regions; } \\
\text { IV.2. fractal analysis, Minkowski functionals, wavelet transform and Laplacian transforms of Gaussian- } \\
\text { filtered images for the abnormal regions. }\end{array}$ \\
\hline
\end{tabular}

ADC, apparent diffusion coefficient; FLAIR, fluid-attenuated inversion recovery; SWI, susceptibility-weighted imaging.

remaining feature subset more than removing any other feature. The iFIBE algorithm iterates between adding and removing features, until no more features can be added or removed to increase the AUC. Using our iFIBE feature selector ${ }^{43} 91$ with Gaussian-kernel SVM, ${ }^{87} 88$ we selected 12 texture features out of $\sim 200$ features for deformable brain MRI registration ${ }^{42}{ }^{43}$; ; we selected 7 from $>300000$ features for histology-based tumour staging $(\mathrm{AUC}=0.96)$ and grading (AUC=0.83), ${ }^{91}$ from 83 patients; we selected 53 features among $>2000$ brain MRI features for classifying bipolar disorder, major depression and normal controls (AUC=0.61), ${ }^{92}$ from 113 subjects; and, we selected 69 from $>2000$ brain MRI features for diagnosing schizophrenia (AUC=0.75) ${ }^{93}$ from 124 subjects.

These MRI features and the SVM/RF prediction models are commonly used radiomics features and models for predicting brain disease progression and outcomes. The use of SVM and RF is because of their wide use in radiomics to predict brain disease progression and outcomes, and also because they are the baseline model on which we built the iFIBE feature selection algorithm (ie, our iFIBE feature selection is at the best performance when coupled with SVM and RF). Using some of these MRI features in prediction models has led to $0.6-1.0$ sensitivity/0.76-0.99 specificity classifying original or metastatic brain tumor, ${ }^{96} 970.77-0.93$ sensitivity/0.76-0.92 specificity predicting good/poor outcomes after acute brain haemorrhage, ${ }^{98} 990.91-0.94$ sensitivity/0.91-1.0 specificity grading brain tumor ${ }^{100} 0.86$ sensitivity/0.92 specificity predicting psychomotor outcome in preterm neonates, ${ }^{101}$ and $0.71-0.82$ sensitivity/0.68-0.92 specificity predicting multiple sclerosis worsening or not ${ }^{102}$ or predicting relapsing-remitting multiple sclerosis (MS) patients. ${ }^{103}$

Other clinical features include patient sex, the extent of PWB (left/right/bilateral), age at first MRI, the extent of brain involvement and EEG report (normal/not). Our hypothesis is that combining these clinical variables with MRI features will improve the sensitivity and specificity of outcome prediction compared with using MRI features alone. Our feature selection algorithm will be used to select the subset of clinical and MRI features that together best predicts the outcome.

\section{Deep learning}

Deep learning convolutional neural network (CNN) takes the whole image or image patches as input for outcome prediction. We will use the transfer learning strategy, ${ }^{104}$ using the pretrained 2D CNN models that were trained in the ImageNet database (>14 million natural images). ${ }^{105-107}$ The big data-powered pretrained CNN model will be refined in our SWS cohort, on 2D slices that contain the abnormal regions. This transfer learning strategy is widely used to enable and stabilise deep learning in small sample studies. Successful applications included imagebased classification and outcome prediction in 2D chest X-ray images, ${ }^{104108}$ 2D retina images, ${ }^{109-112}$ 2D histological images $^{113-118}$ and recently in 3D brain MRI for tumour classification. ${ }^{11}$ Prediction results on various slices of the same patient can be combined through majority voting into a final prediction. ${ }^{116}$ This plan avoids training a 3D CNN directly on our data, where the sample size may lead to overfitting. The accuracy of deep learning outcome prediction will also be quantified by sensitivity, specificity and AUC in fivefold cross-validations and be compared with those metrics by the traditional machine learning.

The transfer learning strategy has been commonly used to deal with small sample problems. For 2D images, we can initialise with the benchmark artificial intelligence (AI) model from imageNet, and refine the model by samples in a specific task, for each specific AI study. ${ }^{104}$ The benchmark AI model can initialise studies (1) across 
sample size: trained in $>14$ million images in ImageNet, it can initialise other AI studies with only hundreds to thousands of samples ${ }^{104114119}$; (2) across tasks: trained to recognise natural objects (eg, cats, dogs, balloons, etc), it can initialise clinical AI tasks, such as disease diagnosis, ${ }^{104110111113115120}$ severity evaluation, ${ }^{109} 117118$ treatment evaluation ${ }^{3}$ and prediction of prognosis ${ }^{108} 112114121$ and (3) across imaging modalities: trained in cameraacquired natural images, it can initialise studies involving chest X-ray images, ${ }_{104} 108$ retina images ${ }^{110} 111$ and histological images. ${ }^{113} 114119$ In these Nature, Cell, Lancet, JAMA, PNAS studies, initialisation with the benchmark model and refinement with samples in each specific AI task has led superior accuracies than training from scratch in specific tasks. ${ }^{104122}$ In 3D medical images, we just trained a 3D benchmark AI model from 16705 brain MRIs across the lifespan for predicting the continuously valued age. ${ }^{123124}$ This offers one of the first benchmark AI models that can be transferred to small sample size studies such as in this SWS case. We will test the prediction accuracy in SWS with and without transferring the benchmark model.

\section{Integrate MRI and non-MRI information}

We hypothesise that such integration can more accurately predict outcomes than using MRI alone. In traditional machine learning models, clinical features can be concatenated into the feature vector for each patient. The remaining steps (classification, feature selection and cross-validation) stay the same. In deep learning models, clinical features can be added into each feature layer in the $\mathrm{CNN}$ architecture, ${ }^{125}$ and the rest is the same. In both cases, the learning process will automatically parse the interaction between MRI and clinical information and find the best combination. We will compare the sensitivity, specificity and AUC between the traditional and deep learning models after combining MRI and clinical information.

\section{Expected sample size and power analysis}

From clinical registries, we have identified 98 patients (62 from KKI and 36 from $\mathrm{BCH}$ ) with brain MRIs before 2 years of age, of which 63 (42 from KKI and 21 from BCH) had the first MRI before the onset of seizure. We expect a more thorough search of clinical registries and ICDbased hospital-wide search can bring us to the expected 80 sample size for infants who had the first MRI before the first onset of seizure. This sample size will be $>5$ times bigger than most existing presymptomatic studies for SWS.

In the single-variate analysis, with power $=0.8$ and alpha $=0.05$, correlating a predictive variable and the neuroscore outcome variable will need 194, 85, 47, 29 subjects to call significance if the correlation is $0.2,0.3$, $0.4,0.5$. Between patient groups presenting and not presenting seizure symptoms by 2 years of age, we need 393, 99 or 63 patients to call significant group differences if a feature has a shared SD between two groups and the difference in this feature between two groups is $20 \%, 40 \%$,or $50 \%$ of the mean of one group. With our expected sample size $(\mathrm{BCH}+\mathrm{KKI})$, we will be able to call a predictive variable significantly associated with outcome if the correlation is greater than 0.41 , and the group difference is more than $50 \%$ of the mean in one group.

In multivariate machine learning, our expected sample size of 80 will allow our algorithm to select up to eight MRI and clinical variables for outcome prediction, according to the 'rule of 10' in multivariate ML prediction. ${ }^{126-128}$ Our algorithm typically selects 5-10 variables from hundreds of variables extracted from MRI and clinical variabels. ${ }^{11} 14254849$ Thus, overfitting is not a big concern in this multisite MRI data for SWS.

\section{Patient and public involvement}

Patient advocacy groups will be involved in the distribution of the summary of the findings after publication of results. The development of the research question was informed by the unmet needs determined during the Sturge-Weber Foundation Clinical Care Network conference in 2018. Patients or the public were not involved in the design, or conduct, or reporting or dissemination plans of our research.

\section{DISCUSSION AND DESSEMINATION}

Secondary use of hospital-hosted data can be time-efficient and cost-efficient. This efficiency is further amplified in rare diseases. A typical clinical trial often requires significant funding and years to collect data from single digits or dozens of patients with SWS. Our study protocol, on the other than, plans to retrospectively collect presymptomatic data for $\sim 80$ patients with SWS who had neuroimaging under 2 years of age and were cared in $\mathrm{BCH}$ and KKI, two large nationwide centres for SWS care, including presymptomatic SWS care.

The importance of identifying reliable biomarkers for early diagnosis of SWS relies on the potential for presymptomatic intervention impacting neurological outcomes of SWS. The first goal of this study is to further elucidate the pathogenesis of early epileptogenesis and brain injury in SWS by quantifying and comparing neuroimaging patterns, which reflect the integrity and reorganisation of neural networks. Comparing brain MRIs in patients with SWS with or without seizures after the first MRI will identify injury patterns that may aid early diagnosis of SWS brain involvement, and guide treatment to improve the prognosis of those at high risk for seizures before 2 years of age and subsequent cognitive and neurologic impairment.

SWS is a developmental disorder and the epileptogenesis mechanism during early ages will likely differ from the mechanisms in the mature brain. Prior studies have repeatedly associated early age of seizure onset in SWS with worse neurologic outcome, more severe epilepsy and worse cognitive outcome. ${ }^{6} 48129$ Thus, delaying or preventing seizure onset in SWS has become a main 
treatment goal driving recent efforts to enhance early diagnosis of brain involvement. ${ }^{9}$ Standard imaging techniques and assessments result in only about a $25 \%$ sensitivity in early imaging ${ }^{130}$ resulting in the loss of opportunity to intervene early in many children and slowing efforts to determine the most effective presymptomatic treatment. The proposed quantitative and objective study may improve early diagnosis. More infants with SWS brain involvement may benefit from presymptomatic studies, anticipatory guidance and close monitoring for neurologic and developmental impairments. Zallman et al showed that infants with PWBs and evidence of SWS brain involvement on imaging were more likely to receive appropriate early care, compared with those who were not diagnosed before the onset of seizures. ${ }^{130}$

SWS is a spectrum disorder with a well-identified pathological process allowing precise diagnosis, and it is an excellent model for LSM to quantify neuroanatomic basis of outcomes. The brain involvement ranging in extent from single lobe (usually occipital) to extensive bilateral brain involvement of both hemispheres. ${ }^{131}$ As a result, the most important factor determining the neurologic and cognitive outcome, other than the age of seizure onset, is the extent of brain involvement. The greater extent of SWS brain involvement is correlated with earlier age of seizure onset. ${ }^{131-133}$ Early age of seizure onset, particularly less than a year of age, has been associated with a significant impact on intelligence quotient (IQ) ${ }^{48}$ worse hemiparesis ${ }^{129}$ and lower cognitive function quality of life. ${ }^{28}$ Therefore, the full extent of SWS brain involvement, as determined by later MRI imaging with contrast and appropriate imaging, after a year of age, is needed; early imaging, even when abnormal, can underestimate the extent of brain involvement. The extent of brain involvement (on imaging after a year of age) will be an important clinical factor in the AI analysis.

Adding clinical variables to neuroimaging is important. Males are associated with worse cognitive outcomes, and with a greater risk of suicidality in SWS. ${ }^{134} 135$ Whether gender impacts the early evolution of SWS as manifested in neuroimaging remains to be studied. A family history of seizures or epilepsy is associated with an early age of seizure onset in SWS. ${ }^{136}$ This suggests that genetic factors, beyond the underlying somatic mutation in GNAQ have a role in determining the timing of seizure onset in SWS. ${ }^{1}$ The extent of skin involvement with PBW may also be a prognostic factor. A greater extent of PWB (bilateral vs unilateral/none) is associated with worse neurologic status. ${ }^{137}$ Bilateral skin involvement is associated with bilateral brain involvement. Therefore, the extent of skin involvement is to some extent a proxy for the extent of brain involvement that impacts the neurologic outcome. However, it has also been shown that patients with no PWB present later and have a better outcome than patients with a similar extent of brain involvement. ${ }^{138} 139$ This observation suggests that more extensive SWS (in terms of more structures (brain, skin and/or eye) involved is in some way different with regards to the evolution of brain injury, from less extensive SWS (brain only) involvement. Gender, family history of epilepsy and the extent of skin involvement are, therefore, also important predictive clinical factors to include in the AI neuroimaging modelling and analysis.

During early stage SWS, the extent of neuroimaging alteration is similar to that of other small diffuse lesions; our sample size is similar to other published datasets (including chronic stroke lesions ${ }^{140141}$ and multiple sclerosis) ${ }^{142-144}$ Identificatication of biomarkers is a complex process but has proved to result in rapid bench-to-bedside translation in many other pathological conditions. The identification of reliable presymptomatic biomarkers will ultimately be used to predict response to therapies and influence 'go' or 'no go' decisions before advancing clinical trials for SWS.

This protocol describes a multicentre effort to validate emerging techniques in a rare potentially devastating disease. The centres involved have the expertise and the combined number of cases, indicating the strong feasibility of this study. The results will benefit future prospective trials.

\section{Ethics and dissemination}

The Institutional Review Boards at Kennedy Krieger Institute and Boston Children's Hospital approval have been obtained at each site to retrospectively study this data. Results will be disseminated by presentations, publication and sharing of algorithms generated.

\section{Author affiliations}

${ }^{1}$ Department of Neurology and Developmental Medicine, Hugo Moser Research Institute, Baltimore, Maryland, USA

${ }^{2}$ Department of Neurology and Pediatrics, Kennedy Krieger Institute, Baltimore, MD, USA

${ }^{3}$ Department of Neurology, Division of Epilepsy, Harvard Medical School, Boston, Massachusetts, USA

${ }^{4}$ Neuroradiology, Johns Hopkins School of Medicine, Baltimore, Maryland, USA ${ }^{5}$ Department of Neurology and Pediatrics, Johns Hopkins School of Medicine, Baltimore, Maryland, USA

${ }^{6}$ Fetal-Neonatal Neuroimaging and Developmental Science Center, Boston Children's Hospital, Boston, Massachusetts, USA

${ }^{7}$ Computational Health Informatics Program, Boston Children's Hospital, Boston, Massachusetts, USA

${ }^{8}$ Department of Radiology, Boston Children's Hospital; Harvard Medical School, Boston, MA, USA

Collaborators BCH-KKI SWS Pre-symptomatic Biomarker Working Group members include: Mustafa Sahin, MD, Boston Children's Hospital. P. Ellen Grant, MD, Boston Children's Hospital. Katrina Boyer, PhD, Boston Children's Hospital. Masanori Takeoka, MD, Boston Children's Hospital. Bernard Cohen MD, Johns Hopkins Dermatology. Joshua Ewen MD, Kennedy Krieger Institute Epilepsy. Eric Kossoff MD, Johns Hopkins Child Neurology and Epilepsy. Teressa Garcia Reidy, MS, OTR/L, Kennedy Krieger Institute Occupational Therapy. Stacy Suskauer MD, Kennedy Krieger Institute Medical Rehabilitation. Andrew Zabel PhD, Kennedy Krieger Institute Neuropsychology.

Contributors Study design: ARLP, AMC, YO. Data acquisition: PV, ARLP, DDML, AMC, YO. Data analysis plan: ARLP, DDML, AMC, YO. Paper drafting and editing: PV, ARLP, DDML, AMC, YO.

Funding Work leading to this proposal was partly funded by the Celebrate Hope Foundation (Comi), Harvard Medical School/ Boston Children's Hospital Faculty Career Development Award (Ou), St. Baldrick Foundation (Ou), and NIH R03 HD091464 (Ou). Application to further NIH funding is under review. 
Competing interests None declared.

Patient and public involvement Patients and/or the public were not involved in the design, or conduct, or reporting, or dissemination plans of this research.

\section{Patient consent for publication Not applicable.}

Provenance and peer review Not commissioned; externally peer reviewed.

Data availability statement Retrospective mining clinical data are approved by IRBs in our institutions, but sharing these datasets needs further legal and ethical approval that will delay or prohibit the sharing process. Data transfer agreement to a third party is possible upon reasonable request to the corresponding authors, and upon the approval from our institutions and the third-party institution.

Open access This is an open access article distributed in accordance with the Creative Commons Attribution Non Commercial (CC BY-NC 4.0) license, which permits others to distribute, remix, adapt, build upon this work non-commercially, and license their derivative works on different terms, provided the original work is properly cited, appropriate credit is given, any changes made indicated, and the use is non-commercial. See: http://creativecommons.org/licenses/by-nc/4.0/.

ORCID iD

Pooja Vedmurthy http://orcid.org/0000-0001-9106-0567

\section{REFERENCES}

1 Shirley MD, Tang H, Gallione CJ, et al. Sturge-Weber Syndrome and Port-Wine Stains Caused by Somatic Mutation in GNAQ. N Engl J Med Overseas Ed 2013;368:1971-9.

2 Jacobs $\mathrm{AH}$, Walton $\mathrm{RG}$. The incidence of birthmarks in the neonate. Pediatrics 1976;58:218-22.

3 Comi AM. Update on Sturge-Weber syndrome: diagnosis, treatment, quantitative measures, and controversies. Lymphat Res Biol 2007;5:257-64.

4 De la Torre AJ, Luat AF, Juhász C, et al. A multidisciplinary consensus for clinical care and research needs for Sturge-Weber syndrome. Pediatr Neurol 2018;84:11-20.

5 Luat AF, Juhász C, Loeb JA, et al. Neurological complications of Sturge-Weber syndrome: current status and unmet needs. Pediatr Neurol 2019:98:31-8.

6 Sujansky E, Conradi S. Sturge-Weber syndrome: age of onset of seizures and glaucoma and the prognosis for affected children. $J$ Child Neurol 1995;10:49-58.

7 Sujansky E, Conradi S. Outcome of Sturge-Weber syndrome in 52 adults. Am J Med Genet 1995;57:35-45.

8 Glauser TA, Mitchell WG, Weinstock A, et al. Pharmacokinetics of levetiracetam in infants and young children with epilepsy. Epilepsia 2007;48:1117-22.

9 Day AM, Hammill AM, Juhász C, et al. Hypothesis: presymptomatic treatment of Sturge-Weber syndrome with aspirin and antiepileptic drugs may delay seizure onset. Pediatr Neurol 2019;90:8-12.

10 Juhász C. Predicting and preventing epilepsy in Sturge-Weber syndrome? Pediatr Neurol Briefs 2016;30:43.

11 Luat AF, Behen ME, Chugani HT, et al. Cognitive and motor outcomes in children with unilateral Sturge-Weber syndrome: effect of age at seizure onset and side of brain involvement. Epilepsy Behav 2018;80:202-7.

12 Bar C, Pedespan J-M, Boccara O, et al. Early magnetic resonance imaging to detect presymptomatic leptomeningeal angioma in children with suspected Sturge-Weber syndrome. Dev Med Child Neurol 2020;62:227-33.

13 Juhász C, Hu J, Xuan Y, et al. Imaging increased glutamate in children with Sturge-Weber syndrome: association with epilepsy severity. Epilepsy Res 2016;122:66-72.

14 Bosnyák E, Behen ME, Guy WC, et al. Predictors of cognitive functions in children with Sturge-Weber syndrome: a longitudinal study. Pediatr Neurol 2016;61:38-45.

15 Kobylecki C, Jones M, Williams T, et al. Reversible increases in cortical diffusion-weighted Mr signal in a patient with Sturge-Weber syndrome and subacute hemiplegia. J Neurol 2011;258:2095-6.

16 Jülich K, Neuberger I, Sahin M, et al. Yield of emergent neuroimaging in patients with Sturge-Weber syndrome presenting with acute neurologic symptoms. J Child Neurol 2019;34:17-21.

17 Wu J, Tarabishy B, Hu J, et al. Cortical calcification in Sturge-Weber syndrome on MRI-SWI: relation to brain perfusion status and seizure severity. J Magn Reson Imaging 2011;34:791-8.

18 Dutkiewicz A-S, Ezzedine K, Mazereeuw-Hautier J, et al. A prospective study of risk for Sturge-Weber syndrome in children with upper facial port-wine stain. J Am Acad Dermatol 2015;72:473-80.

19 Arulrajah S, Ertan G, M Comi A, et al. Mri with diffusion-weighted imaging in children and young adults with simultaneous supraand infratentorial manifestations of Sturge-Weber syndrome. Neuroradiol 2010;37:51-9.

$20 \mathrm{Kim} \mathrm{J}-\mathrm{A}$, Jeong J-W, Behen ME, et al. Metabolic correlates of cognitive function in children with unilateral Sturge-Weber syndrome: evidence for regional functional reorganization and crowding. Hum Brain Mapp 2018;39:1596-606.

21 Pinto AL, Chen L, Friedman R, et al. Sturge-Weber Syndrome: Brain Magnetic Resonance Imaging and Neuropathology Findings. Pediatr Neurol 2016;58:25-30.

22 Aerts HJWL, Velazquez ER, Leijenaar RTH, et al. Decoding tumour phenotype by noninvasive imaging using a quantitative radiomics approach. Nat Commun 2014;5:4006.

23 Lambin P, Rios-Velazquez E, Leijenaar R, et al. Radiomics: extracting more information from medical images using advanced feature analysis. Eur J Cancer 2012;48:441-6.

24 Winzeck S, Hakim A, McKinley R, et al. Isles 2016 and 2017-Benchmarking ischemic stroke lesion outcome prediction based on multispectral MRI. Front Neurol 2018;9:679.

25 Rodriguez Gutierrez D, Manita M, Jaspan T, et al. Serial Mr diffusion to predict treatment response in high-grade pediatric brain tumors: a comparison of regional and voxel-based diffusion change metrics. Neuro Oncol 2013;15:981-9.

26 Uddin LQ, Dajani DR, Voorhies W, et al. Progress and roadblocks in the search for brain-based biomarkers of autism and attentiondeficit/hyperactivity disorder. Trans/ Psychiatry 2017;7:e1218.

27 Weiss RJ, Bates SV, Song Ya'nan, et al. Mining multi-site clinica data to develop machine learning MRI biomarkers: application to neonatal hypoxic ischemic encephalopathy. $J$ Transl Med 2019;17:385.

28 Hatfield LA, Crone NE, Kossoff EH, et al. Quantitative EEG asymmetry correlates with clinical severity in unilateral SturgeWeber syndrome. Epilepsia 2007;48:191-5.

29 Lin DDM, Barker PB, Hatfield LA, et al. Dynamic Mr perfusion and proton MR spectroscopic imaging in Sturge-Weber syndrome: correlation with neurological symptoms. J Magn Reson Imaging 2006;24:274-81.

30 Kelley TM, Hatfield LA, Lin DDM, et al. Quantitative analysis of cerebral cortical atrophy and correlation with clinical severity in unilateral Sturge-Weber syndrome. J Child Neurol 2005;20:867-70.

31 Kavanaugh B, Sreenivasan A, Bachur C, et al. [Formula: see text] Intellectual and adaptive functioning in Sturge-Weber Syndrome. Child Neuropsychol 2016;22:635-48.

32 Pienaar R, Rannou N, Bernal J, et al. ChRIS--A web-based neuroimaging and informatics system for collecting, organizing, processing, visualizing and sharing of medical data. Annu Int Conf IEEE Eng Med Biol Soc 2015;2015:206-9.

33 Ou Y, Zöllei L, Retzepi K, et al. Using clinically acquired MRI to construct age-specific ADC atlases: quantifying spatiotemporal ADC changes from birth to 6-year old. Hum Brain Mapp 2017;38:3052-68.

34 Murphy SN, Weber G, Mendis M, et al. Serving the enterprise and beyond with informatics for integrating biology and the bedside (i2b2). J Am Med Inform Assoc 2010;17:124-30.

35 Patridge EF, Bardyn TP. Research electronic data capture (REDCap). J Med Libr Assoc 2018;106:142-4.

36 Tustison NJ, Avants BB, Cook PA, et al. N4ITK: improved N3 bias correction. IEEE Trans Med Imaging 2010;29:1310-20.

37 Ou Y, Zöllei L, Da X, et al. Field of view normalization in multi-site brain MRI. Neuroinformatics 2018;16:431-44.

38 Srinivasan D, Erus G, Doshi J, et al. A comparison of Freesurfer and multi-atlas MUSE for brain anatomy segmentation: findings about size and age bias, and inter-scanner stability in multi-site aging studies. Neuroimage 2020;223:117248.

39 Doshi J, Erus G, Ou Y, et al. Muse: MUlti-atlas region segmentation utilizing ensembles of registration algorithms and parameters, and locally optimal atlas selection. Neuroimage 2016;127:186-95.

40 Sotardi S, Gollub RL, Bates SV, et al. Voxelwise and regional brain apparent diffusion coefficient changes on MRI from birth to 6 years of age. Radiology 2021;298:415-24.

41 Morton SU, Vyas R, Gagoski B, et al. Maternal dietary intake of omega-3 fatty acids correlates positively with regional brain volumes in 1-month-old term infants. Cereb Cortex 2020;30:2057-69.

$42 \mathrm{Ou}$ Y, Akbari H, Bilello M, et al. Comparative evaluation of registration algorithms in different brain databases with varying difficulty: results and insights. IEEE Trans Med Imaging 2014;33:2039-65. 
43 Ou Y, Sotiras A, Paragios N, et al. DRAMMS: deformable registration via attribute matching and mutual-saliency weighting. Med Image Anal 2011;15:622-39.

44 Jenkinson M, Beckmann CF, Behrens TEJ, et al. Fsl. Neuroimage 2012;62:782-90.

45 Fortin J-P, Parker D, Tunç B, et al. Harmonization of multi-site diffusion tensor imaging data. Neuroimage 2017;161:149-70.

46 Johnson WE, Li C, Rabinovic A. Adjusting batch effects in microarray expression data using empirical Bayes methods. Biostatistics 2007;8:118-27.

47 Martí-Bonmatí L, Menor F, Mulas F. The Sturge-Weber syndrome: correlation between the clinical status and radiological CT and MRI findings. Childs Nerv Syst 1993;9:107-9.

48 Juhasz C, Lai C, Behen ME, et al. White matter volume as a major predictor of cognitive function in Sturge-Weber syndrome. Arch Neurol 2007;64:1169-74

49 Arigliani M, Bravar G, Crichiutti G, et al. A cerebral infarction in a girl with Sturge-Weber syndrome. Pediatr Neurol 2016;64:99-100.

50 Adamsbaum C, Pinton F, Rolland Y, et al. Accelerated myelination in early Sturge-Weber syndrome: MRI-SPECT correlations. Pediatr Radiol 1996;26:759-62.

51 George U, Rathore S, Nittala P. Mr demonstration of accelerated myelination in early sturge Weber syndrome. Neurol India 2010;58:336.

52 Moritani T, Kim J, Sato Y, et al. Abnormal hypermyelination in a neonate with Sturge-Weber syndrome demonstrated on diffusiontensor imaging. J Magn Reson Imaging 2008;27:617-20.

53 Pilli VK, Behen ME, Hu J, et al. Clinical and metabolic correlates of cerebral calcifications in Sturge-Weber syndrome. Dev Med Child Neurol 2017;59:952-8

54 Pilli VK, Chugani HT, Juhász C. Enlargement of deep medullary veins during the early clinical course of Sturge-Weber syndrome. Neurology 2017;88:103-5.

55 Kaseka ML, Bitton JY, Décarie J-C, et al. Predictive factors for epilepsy in pediatric patients with Sturge-Weber syndrome. Pediatr Neurol 2016;64:52-8

56 John F, Maqbool M, Jeong J-W, et al. Deep cerebral vein expansion with metabolic and neurocognitive recovery in Sturge-Weber syndrome. Ann Clin Transl Neurol 2018;5:502-6.

57 Juhász C, Haacke EM, Hu J, et al. Multimodality imaging of cortical and white matter abnormalities in Sturge-Weber syndrome. AJNR Am J Neuroradiol 2007;28:900-6.

58 Alkonyi B, Chugani HT, Behen $\mathrm{M}$, et al. The role of the thalamus in neuro-cognitive dysfunction in early unilateral hemispheric injury: a multimodality imaging study of children with Sturge-Weber syndrome. Eur J Paediatr Neurol 2010;14:425-33.

59 Pinto ALR, Ou Y, Sahin M, et al. Quantitative apparent diffusion coefficient mapping may predict seizure onset in children with Sturge-Weber syndrome. Pediatr Neurol 2018;84:32-8.

60 Kamson DO, Juhász C, Shin J, et al. Patterns of structura reorganization of the corticospinal tract in children with SturgeWeber syndrome. Pediatr Neurol 2014;50:337-42.

61 Jeong J-won, Tiwari VN, Shin J, et al. Assessment of brain damage and plasticity in the visual system due to early occipital lesion: comparison of FDG-PET with diffusion MRI tractography. J Magn Reson Imaging 2015;41:431-8.

62 Alkonyi B, Govindan RM, Chugani HT, et al. Focal white matter abnormalities related to neurocognitive dysfunction: an objective diffusion tensor imaging study of children with Sturge-Weber syndrome. Pediatr Res 2011;69:74-9.

63 Guimond A, Meunier J, Thirion J-P. Average brain models: a convergence study. Computer Vision and Image Understanding 2000;77:192-210.

64 Fonov V, Evans AC, Botteron K, et al. Unbiased average age-appropriate atlases for pediatric studies. Neuroimage 2011;54:313-27.

65 O'Muircheartaigh J, Robinson EC, Pietsch M, et al. Modelling brain development to detect white matter injury in term and preterm born neonates. Brain 2020;143:467-79.

66 Buschman TJ, Siegel M, Roy JE, et al. Neural substrates of cognitive capacity limitations. Proc Natl Acad Sci U S A 2011;108:11252-5.

67 Pagonabarraga J, Kulisevsky J, Strafella AP, et al. Apathy in Parkinson's disease: clinical features, neural substrates, diagnosis, and treatment. Lancet Neurol 2015;14:518-31.

68 Bates E, Wilson SM, Saygin AP, et al. Voxel-Based lesion-symptom mapping. Nat Neurosci 2003;6:448-50.

69 Rorden C, Karnath H-O, Bonilha L. Improving lesion-symptom mapping. J Cogn Neurosci 2007;19:1081-8.
70 Zhang Y, Kimberg DY, Coslett HB, et al. Multivariate lesionsymptom mapping using support vector regression. Hum Brain Mapp 2014;35:5861-76.

71 DeMarco AT, Turkeltaub PE. A multivariate lesion symptom mapping toolbox and examination of lesion-volume biases and correction methods in lesion-symptom mapping. Hum Brain Mapp 2018;39:4169-82.

72 Fox MD. Mapping symptoms to brain networks with the human connectome. N Engl J Med 2018;379:2237-45.

73 Gleichgerrcht E, Fridriksson J, Rorden C, et al. Connectomebased lesion-symptom mapping (CLSM): a novel approach to map neurological function. Neuroimage Clin 2017;16:461-7.

74 Yourganov G, Fridriksson J, Rorden C, et al. Multivariate Connectome-Based symptom mapping in post-stroke patients: networks supporting language and speech. J Neurosci 2016;36:6668-79.

75 Sporns O. The human connectome: a complex network. Ann N Y Acad Sci 2011;1224:109-25.

76 Van Essen DC, Smith SM, Barch DM, et al. The WU-Minn human connectome project: an overview. Neuroimage 2013;80:62-79.

77 van den Heuvel MP, Sporns O. Rich-club organization of the human connectome. J Neurosci 2011;31:15775-86.

78 Liu TT, Achrol AS, Mitchell LA, et al. Computational identification of tumor anatomic location associated with survival in 2 large cohorts of human primary glioblastomas. AJNR Am J Neuroradiol 2016;37:621-8.

79 Hope TMH, Leff AP, Price CJ. Predicting language outcomes after stroke: is structural disconnection a useful predictor? Neuroimage Clin 2018;19:22-9.

80 Xing S, Lacey EH, Skipper-Kallal LM, et al. Right hemisphere grey matter structure and language outcomes in chronic left hemisphere stroke. Brain 2016;139:227-41.

81 Mirman D, Chen Q, Zhang Y, et al. Neural organization of spoken language revealed by lesion-symptom mapping. Nat Commun 2015;6:6762.

82 Karnath $\mathrm{H}-\mathrm{O}$, Rennig J. Investigating structure and function in the healthy human brain: validity of acute versus chronic lesionsymptom mapping. Brain Struct Funct 2017;222:2059-70.

83 Bonilha L, Hillis AE, Hickok G, et al. Temporal lobe networks supporting the comprehension of spoken words. Brain 2017;140:2370-80.

84 Roswandowitz C, Kappes C, Obrig H, et al. Obligatory and facultative brain regions for voice-identity recognition. Brain 2018;141:234-47.

85 Liu J, Wang C, Diao Q, et al. Connection disruption underlying attention deficit in subcortical stroke. Radiology 2018;288:186-94.

86 Meyer S, Kessner SS, Cheng B, et al. Voxel-Based lesion-symptom mapping of stroke lesions underlying somatosensory deficits. Neuroimage Clin 2016;10:257-66.

87 Cortes C, Vapnik V. Support-vector networks. Mach Learn 1995;20:273-97.

88 Chang C-C, Lin C-J. LIBSVM: a library for support vector machines. ACM Trans Intell Syst Technol 2011;2:Article 27.

89 Breiman L. Random forests. Mach Learn 2001;45:5-32.

90 Hemant I, Udaya BK, Eugene HB. Random survival forests. The Annals of Applied Statistics 2008;2:841-60.

91 Ou Y, Shen D, Zeng J, et al. Sampling the spatial patterns of cancer: optimized biopsy procedures for estimating prostate cancer volume and Gleason score. Med Image Anal 2009;13:609-20.

92 Serpa MH, Ou Y, Schaufelberger MS, et al. Neuroanatomical classification in a population-based sample of psychotic major depression and bipolar I disorder with 1 year of diagnostic stability. Biomed Res Int 2014;2014:1-9.

93 Zanetti MV, Schaufelberger MS, Doshi J, et al. Neuroanatomical pattern classification in a population-based sample of first-episode schizophrenia. Prog Neuropsychopharmacol Biol Psychiatry 2013;43:116-25.

$94 \mathrm{Da}$ X, Toledo JB, Zee J, et al. Integration and relative value of biomarkers for prediction of $\mathrm{MCl}$ to $\mathrm{AD}$ progression: spatial patterns of brain atrophy, cognitive scores, APOE genotype and CSF biomarkers. Neuroimage Clin 2014:4:164-73.

$95 \mathrm{Ou}$ Y, Weinstein SP, Conant EF, et al. Deformable registration for quantifying longitudinal tumor changes during neoadjuvant chemotherapy. Magn Reson Med 2015;73:2343-56.

96 Chen C, Ou X, Wang J, et al. Radiomics-Based machine learning in differentiation between glioblastoma and metastatic brain tumors. Front Oncol 2019;9:806.

97 Artzi M, Bressler I, Ben Bashat D. Brain metastasis and subtypes using radiomics analysis. J Magn Reson Imaging 2019;50:519-28. 
98 Nawabi J, Kniep H, Elsayed S, et al. Imaging-Based outcome prediction of acute intracerebral hemorrhage. Trans/ Stroke Res 2021;12:958-67.

$99 \mathrm{Xu} \mathrm{X}$, Zhang J, Yang K, et al. Prognostic prediction of hypertensive intracerebral hemorrhage using CT radiomics and machine learning Brain Behav 2021;11:e02085.

100 Chu H, Lin X, He J, et al. Value of MRI Radiomics based on enhanced T1WI images in prediction of meningiomas grade. Acad Radiol 2021;28:687-93.

101 Shin Y, Nam Y, Shin T, et al. Brain MRI radiomics analysis may predict poor psychomotor outcome in preterm neonates. Eur Radiol 2021;31:6147-55

102 Zhao Y, Healy BC, Rotstein D, et al. Exploration of machine learning techniques in predicting multiple sclerosis disease course. PLOS One 2017;12:e0174866.

103 Doyle A, Precup D, Arnold D. Predicting future disease activity and treatment responders for multiple sclerosis patients using a Bag-ofLesions brain representation. Lecture Notes in Computer Science 2017:186-94.

104 Kermany DS, Goldbaum M, Cai W, et al. Identifying medical diagnoses and treatable diseases by image-based deep learning. Cell 2018;172:1122-31.

105 Deng J, Dong W, Socher R. ImageNet: a large-scale hierarchica image database. 2009 IEEE conference on computer vision and pattern recognition 2009:248-55.

106 Krizhevsky A, Sutskever I, Hinton GE. ImageNet classification with deep convolutional neural networks. Commun ACM 2017;60:84-90.

107 Russakovsky O, Deng J, Su H, et al. ImageNet large scale visual recognition challenge. Int $J$ Comput Vis 2015;115:211-52.

108 Toba S, Mitani Y, Yodoya N, et al. Prediction of pulmonary to systemic flow ratio in patients with congenital heart disease using deep Learning-Based analysis of chest radiographs. JAMA Cardiol 2020;5:449-57

109 Burlina PM, Joshi N, Pekala M, et al. Automated grading of age-related macular degeneration from color fundus images using deep Convolutional neural networks. JAMA Ophthalmol 2017;135:1170-6.

110 Gulshan V, Peng L, Coram M, et al. Development and validation of a deep learning algorithm for detection of diabetic retinopathy in retinal fundus Photographs. JAMA 2016;316:2402-10.

111 Ting DSW, Cheung CY-L, Lim G, et al. Development and validation of a deep learning system for diabetic retinopathy and related eye diseases using retinal images from multiethnic populations with diabetes. JAMA 2017;318:2211-23.

112 Poplin R, Varadarajan AV, Blumer K, et al. Prediction of cardiovascular risk factors from retinal fundus Photographs via deep learning. Nat Biomed Eng 2018;2:158-64.

113 Campanella G, Hanna MG, Geneslaw L, et al. Clinical-Grade computational pathology using weakly supervised deep learning on whole slide images. Nat Med 2019;25:1301-9.

114 Coudray N, Ocampo PS, Sakellaropoulos T, et al. Classification and mutation prediction from non-small cell lung cancer histopathology images using deep learning. Nat Med 2018;24:1559-67.

115 Hollon TC, Pandian B, Adapa AR, et al. Near real-time intraoperative brain tumor diagnosis using stimulated Raman histology and deep neural networks. Nat Med 2020;26:52-8.

116 Buda M, AlBadawy EA, Saha A, et al. Deep Radiogenomics of Lower-Grade gliomas: Convolutional neural networks predict tumor genomic subtypes using Mr images. Radiol Artif Intell 2020;2:e180050.

117 Bulten W, Pinckaers H, van Boven $\mathrm{H}$, et al. Automated deeplearning system for Gleason grading of prostate cancer using biopsies: a diagnostic study. Lancet Oncol 2020;21:233-41.

118 Ström P, Kartasalo K, Olsson H, et al. Artificial intelligence for diagnosis and grading of prostate cancer in biopsies: a populationbased, diagnostic study. Lancet Oncol 2020;21:222-32.

119 Kather JN, Pearson AT, Halama N, et al. Deep learning can predict microsatellite instability directly from histology in gastrointestinal cancer. Nat Med 2019;25:1054-6.

120 Esteva A, Kuprel B, Novoa RA, et al. Dermatologist-level classification of skin cancer with deep neural networks. Nature 2017:542:115-8.

121 Mobadersany P, Yousefi S, Amgad M, et al. Predicting cancer outcomes from histology and genomics using convolutional networks. Proc Natl Acad Sci U S A 2018;115:E2970-9.
122 Huh M, Agrawal P, Efros A. What makes ImageNet good for transfer learning? 2016

123 He S, Pereira D, David Perez J, et al. Multi-Channel attention-fusion neural network for brain age estimation: accuracy, generality, and interpretation with 16,705 healthy MRIs across lifespan. Med Image Anal 2021;72:102091.

124 He S, Grant PE, Ou Y. Global-Local transformer for brain age estimation. IEEE Trans Med Imaging 2022;41:213-24.

125 Huang S-C, Pareek A, Seyyedi S, et al. Fusion of medical imaging and electronic health records using deep learning: a systematic review and implementation guidelines. NPJ Digit Med 2020;3:136.

126 Harrell FE, Lee KL, Califf RM, et al. Regression modelling strategies for improved prognostic prediction. Stat Med 1984;3:143-52.

127 Harrell FE, Lee KL, Mark DB. Multivariable prognostic models: issues in developing models, evaluating assumptions and adequacy, and measuring and reducing errors. Stat Med 1996;15:361-87.

128 Riley RD. Correction to: minimum sample size for developing a multivariable prediction model: part II-binary and time-toevent outcomes by Riley RD, Snell Ki, Ensor J, et al. Stat Med 2019;38:5672.

129 Kossoff EH, Ferenc L, Comi AM. An infantile-onset, severe, yet sporadic seizure pattern is common in Sturge-Weber syndrome. Epilepsia 2009;50:2154-7.

130 Zallmann M, Mackay MT, Leventer RJ, et al. Retrospective review of screening for Sturge-Weber syndrome with brain magnetic resonance imaging and electroencephalography in infants with high-risk port-wine stains. Pediatr Dermatol 2018;35:575-81.

131 Bebin EM, Gomez MR. Prognosis in Sturge-Weber disease: comparison of unihemispheric and bihemispheric involvement. $J$ Child Neurol 1988;3:181-4.

132 Alkonyi B, Chugani HT, Karia S, et al. Clinical outcomes in bilateral Sturge-Weber syndrome. Pediatr Neurol 2011;44:443-9.

133 Zabel TA, Reesman J, Wodka EL, et al. Neuropsychological features and risk factors in children with Sturge-Weber syndrome: four case reports. Clin Neuropsychol 2010;24:841-59.

134 Harmon KA, Day AM, Hammill AM, et al. Quality of life in children with Sturge-Weber syndrome. Pediatr Neurol 2019;101:26-32.

135 Sebold AJ, Ahmed AS, Ryan TC, et al. Suicide screening in SturgeWeber syndrome: an important issue in need of further study. Pediatr Neurol 2020;110:80-6.

136 Day AM, McCulloch CE, Hammill AM, et al. Physical and family history variables associated with neurological and cognitive development in Sturge-Weber syndrome. Pediatr Neurol 2019;96:30-6.

137 Dymerska M, Kirkorian AY, Offermann EA, et al. Size of facial portwine birthmark may predict neurologic outcome in Sturge-Weber syndrome. J Pediatr 2017;188:205-9.

138 Comi AM, Fischer R, Kossoff EH. Encephalofacial angiomatosis sparing the occipital lobe and without facial nevus: on the spectrum of Sturge-Weber syndrome variants? J Child Neurol 2003;18:35-8.

139 Aylett SE, Neville BG, Cross JH, et al. Sturge-Weber syndrome: cerebral haemodynamics during seizure activity. Dev Med Child Neurol 1999;41:480-5.

140 Liew S-L, Anglin JM, Banks NW, et al. A large, open source dataset of stroke anatomical brain images and manual lesion segmentations. Sci Data 2018;5:180011.

141 Chen X, You S, Tezcan KC, et al. Unsupervised lesion detection via image restoration with a normative prior. Med Image Anal 2020;64:101713.

142 Carass A, Roy S, Jog A, et al. Longitudinal multiple sclerosis lesion segmentation: resource and challenge. Neuroimage 2017;148:77-102

143 Commowick O, Istace A, Kain M, et al. Objective evaluation of multiple sclerosis lesion segmentation using a data management and processing infrastructure. Sci Rep 2018;8:13650.

144 Karimi D, Dou H, Warfield SK, et al. Deep learning with noisy labels: exploring techniques and remedies in medical image analysis. Med Image Anal 2020;65:101759.

145 Oishi K, Mori S, Donohue PK, et al. Multi-contrast human neonatal brain atlas: application to normal neonate development analysis. Neuroimage 2011;56:8-20. 